\title{
Automated DBS Extraction Prior to Hilic/RP LC-MS/MS Target Screening of Drugs
}

\author{
François Versace · Julien Déglon · Estelle Lauer • \\ Patrice Mangin $\cdot$ Christian Staub
}

Received: 29 October 2012/Revised: 21 November 2012/Accepted: 30 November 2012/Published online: 25 December 2012

(C) Springer-Verlag Berlin Heidelberg 2012

\begin{abstract}
This article describes a rapid LC-MS/MS target screening method based on an automated extraction of $5 \mu \mathrm{L}$ dried blood spots (DBS), two 5 min chromatographic runs on orthogonal phase columns (RP and Hilic) and a data dependent acquisition (DDA) of product ions spectra for the reliable identification of the detected compounds. The extraction step was performed in 2 min by using the LC autosampler itself in 96-well plates. This procedure was evaluated using 22 model compounds frequently encountered in forensic investigations, i.e., cocaine, benzodiazepines, amphetamines, opioids, antidepressants and antipsychotics. These investigations showed that even if the extraction step was reduced to a minimum, the extraction recoveries were satisfactory (median value of $40 \%$ ) and allowed for the detection of the model compounds in their therapeutic ranges, with the exception of morphine. Moreover, the use of two different chromatographic columns broadened the number of screening targets to those that behaved poorly under RP conditions, such as amphetamines or glucuronides, while keeping chromatographic gradients very short. This procedure was applied to 34 authentic postmortem cases. It allowed the detection of $89 \%$ of the compounds that were quantified in the routine procedures and the formal identification of $77 \%$ of the compounds using their product ions spectra. These results were
\end{abstract}

Published in the topical collection Miniaturized and New Featured Planar Chromatography and Related Techniques with guest editor Paweł K. Zarzycki.

F. Versace · J. Déglon · E. Lauer · P. Mangin · C. Staub $(\bowtie)$

Unit of Toxicology, University Center of Legal Medicine,

1 Rue Michel-Servet, 12114 Geneva-Lausanne, Switzerland

e-mail: christian.staub@hcuge.ch

F. Versace

e-mail: francois.versace@unige.ch considered more than satisfactory compared to routine screening alone (GC-MS and LC-DAD, $55 \%$ compound identification). The method described in this article is therefore a powerful approach for a fast, reliable and efficient target screening of drugs in forensic and clinical investigations.

Keywords DBS - Target screening - LC-MS/MS · Automated sample preparation

\section{Introduction}

Drug screening is an important issue in clinical and forensic toxicology that provides essential information for both making a diagnosis and confirmatory quantitative analysis. A screening procedure should ideally enable the detection and identification of any substance of toxicological interest in biological fluids. As the detection capability strongly depends on the matrix, sample preparation and analysis technique, toxicological screening generally consists of a combination of complementary analytical procedures, primarily immunoassays, chromatography and mass spectrometry. This combination gives a precise overview of the substances that were taken [1]. This approach is usually called systematic toxicological analysis (STA). Urine, blood, oral fluid and hair are the most common matrices for forensic STA procedures, each of which providing a different consumption time window [2].

Blood samples (i.e., whole blood, plasma and serum) have a particular value for forensic investigation, as they give information on the acute state of the patient $[2,3]$. However, collecting blood from a living patient is much more invasive than urine and requires medical supervision and particular logistics [4]. Furthermore, in post-mortem 
investigations, volumes of blood may be too scarce to perform any analysis [5].

The use of dried blood spots (DBS) can help because they represent a fast, simple and economical way to collect blood material [6]. A small amount of blood (typically 5-25 $\mu \mathrm{L}$ ), obtained from post-mortem cases or directly after a finger prick on living patients, is spotted onto a cellulose card. Shipment and storage can then be performed without dry ice or refrigeration because most of the analytes are stable at ambient temperature, greatly facilitating handling in this sampling format [7].

Traditionally used for neonatal screening of metabolic disorders, the DBS approach has been extended to different biomedical applications, including therapeutic drug monitoring, clinical and preclinical trials, epidemiological studies and disease surveillance [7]. Although some literature has assessed the potential of DBS for drug screening, especially in doping analysis [8], this alternative sampling method has not yet been implemented for forensic applications.

Gas chromatography coupled to mass spectrometry (GC-MS) is still considered the gold standard for STA [9], but liquid chromatography coupled to mass spectrometry (LC-MS) is now well established as a powerful alternative for screening blood $[3,10]$. The latter method increases the screening capabilities for polar and non-volatile compounds and simplifies sample preparation. Reversed Phase (RP) LC is generally used in screening procedures, as it enables the separation of a wide variety of analytes. Methods have been published to enhance the separation capabilities of LC systems using an orthogonal phases column-switching set-up [11, 12]. Though this approach has high potential, its implementation is relatively complex, which has limited its use in toxicological laboratories.

The coupling of RP HPLC to single MS has been described [13, 14], but tandem MS could greatly improve the selectivity of these screening methods $[15,16]$. In this configuration, identification of unknown compounds is generally based on product ion spectra acquired on lowresolution mass spectrometers, such as tridimensional ion trap (3D IT) or linear ion trap (LIT) instruments. These instruments feature a fast scanning speed in full scan mode and allow $\mathrm{MS}^{\mathrm{n}}$ experiments, which greatly improve the identification capabilities. Using this type of setup, procedures have been published that allow the general unknown screening of drugs [16-18]. Effective multi-target screening applications have also been published [19-21], significantly increasing selectivity and sensitivity due to the concomitant use of multiple reactions monitoring (MRM) acquisition with product ion scans. For this, hybrid systems $\left(\mathrm{QqQ}_{\text {LIT }}\right)$ coupling the advantages of a triple quadrupole platform $(\mathrm{QqQ})$ with a LIT are employed in data-dependent acquisition (DDA) mode [22].
Sample preparation is a crucial step in screening procedures. Indeed, it is essential to remove matrix components that could impair the detection and identification performance without eliminating the compounds of interest. Off-line procedures are typically used for this purpose, including liquid-liquid extraction (LLE) and solid phase extraction (SPE) [3]. Although these procedures are well described, they are tedious and time consuming, which hinder rapid drug screening. Thus, alternatives have been proposed to improve analytical throughput, such as on-line SPE, turbulent flow chromatography (TFC) [23] and diluteand-shoot approaches [24].

Sample preparation should ideally be rapid, simple, online and automated. Recently, our group showed that, besides its well-described benefits from the medical and logistical points of view [25], dried blood spot (DBS) sampling allows for greatly simplifying the extraction procedure without sacrificing excellent analytical performance [26]. The purpose of this work was to propose an automated on-line DBS extraction procedure prior to rapid LC-MS/MS target screening of drugs as a complementary tool for STA. DBS of $5 \mu \mathrm{L}$ were considered in this work, as this is the laboratory standard format for quantitative analysis. The benefits gained through the use of two columns with orthogonal phases were also investigated.

\section{Materials and Methods}

\section{Chemicals and Samples}

All reference compounds and deuterated analogs were purchased at $1,000 \mu \mathrm{g} / \mathrm{mL}$ or $100 \mu \mathrm{g} / \mathrm{mL}$ in methanol (MeOH) or other suitable solvent from Cerilliant (Round Rock, USA) or Lipomed (Arlesheim, Switzerland). Working standard mixtures were prepared by diluting these stock solutions in $\mathrm{MeOH}$ to obtain the concentration of interest. A solution of trimipramine-d3, used as a chromatographic standard (CS), was prepared separately at $10 \mathrm{ng} / \mathrm{mL}$ in $\mathrm{MeOH}$. This solution was used as the extractive solvent. After use, stock and working solutions were stored at $-20{ }^{\circ} \mathrm{C}$.

Both acetonitrile $(\mathrm{ACN})$ and $\mathrm{MeOH}$ were of high-performance chromatographic grade from Merck (Darmstadt, Germany). The $200 \mathrm{mM}$ formate buffer (FB) was prepared from ammonium formate (Fluka). A pH of 3.2 was obtained by adjusting with $1 \%$ aqueous hydrochloric acid (Merck). All human blood, including blank blood, was supplied by the University Center of Legal Medicine (Geneva, Switzerland).

\section{Preparation of Spiked Blood}

Fresh blank whole blood was spiked to the concentrations of interest for 22 model compounds (i.e., cocaine, cocaethylene, 
benzoylecgonine, ecgonine methylester (EME), amphetamine, 3,4-methylenedioxy- $N$-methylamphetamine (MDMA), methamphetamine, morphine, morphine-3-glucuronide (morphineglu), 6-monoacetylmorphine (6-MAM), codeine, methadone, diazepam, 7-aminoclonazepam, midazolam, $\alpha$-hydroxymidazolam (OH-midazolam), zolpidem, amitriptyline, citalopram, trimipramine, clozapine and haloperidol) by adding a suitable volume of the corresponding stock solution to a plastic microtube. The solvent was then evaporated to dryness under a gentle stream of nitrogen, and blood was added to the residue. The spiked blood was then vortexed to ensure the dissolution of the analytes. The DBS obtained from spiked blood were designated as spiked DBS in this work.

\section{Sample Pre-Treatment}

$5 \mu \mathrm{L}$ of real or spiked whole blood was spotted on a filter paper card protein saver 903 from Whatman (Dassel, Germany) using a volumetric micropipette (Eppendorf, Hamburg, Germany). The blood spots were allowed to dry at room temperature for $1 \mathrm{~h}$ and then packed in a sealable plastic bag containing desiccant until analysis. These samples were stored in the dark at ambient temperature.

Discs $6 \mathrm{~mm}$ in diameter that covered the entire DBS were punched out and directly introduced into a 96-well plate. Extraction was performed by the LC autosampler itself as follows. First, $100 \mu \mathrm{L}$ of $\mathrm{MeOH}$ containing the CS was introduced into the desired well by the autosampler syringe. Mixing was produced by dispensing $100 \mu \mathrm{L}$ of air three times in the extraction well through the syringe, and then by aspirating and dispensing $50 \mu \mathrm{L}$ of the well contents twice. This operation lasted $2 \mathrm{~min}$. After rinsing the syringe internally and externally with $\mathrm{ACN}, 5 \mu \mathrm{L}$ of the extract was injected into the chromatographic system.

\section{Dual LC}

The DBS analysis was performed using a LC-MS/MS system consisting of a 5500 QTrap $^{\circledR}\left(\mathrm{QqQ}_{\text {LIT }}\right)$ mass spectrometer equipped with a TurboIon Spray ${ }^{\mathrm{TM}}$ interface (AB Sciex, Concord, Canada) and an Ultimate 3000 RS pump (Dionex, Sunnyvale, CA, USA) as the LC system. Data were acquired and processed using Analyst software (version 1.5.2; AB Sciex, Toronto, Canada).

Two columns were set up in parallel (Fig. 1) to achieve chromatographic separation: a $50 \mathrm{~mm} \times 2.1 \mathrm{~mm}$ i.d., $2.6 \mu \mathrm{m}$ Kinetex RP C18 column and a $50 \mathrm{~mm} \times 2.1 \mathrm{~mm}$ i.d., $2.6 \mu \mathrm{m}$ Kinetex Hilic column (Phenomenex, Torrance, USA). For the RP column, the mobile phase (MP) consisted of a mixture of $\mathrm{H}_{2} \mathrm{O}$ and $\mathrm{ACN}$ set at a flow rate of $0.6 \mathrm{~mL} / \mathrm{min}$, while a mixture of $\mathrm{H}_{2} \mathrm{O}, \mathrm{ACN}$ and $\mathrm{FB}$ at a flow rate of $0.7 \mathrm{~mL} / \mathrm{min}$ was used for the Hilic column. The chromatographic run was divided into two phases, corresponding to the subsequent injections of the same

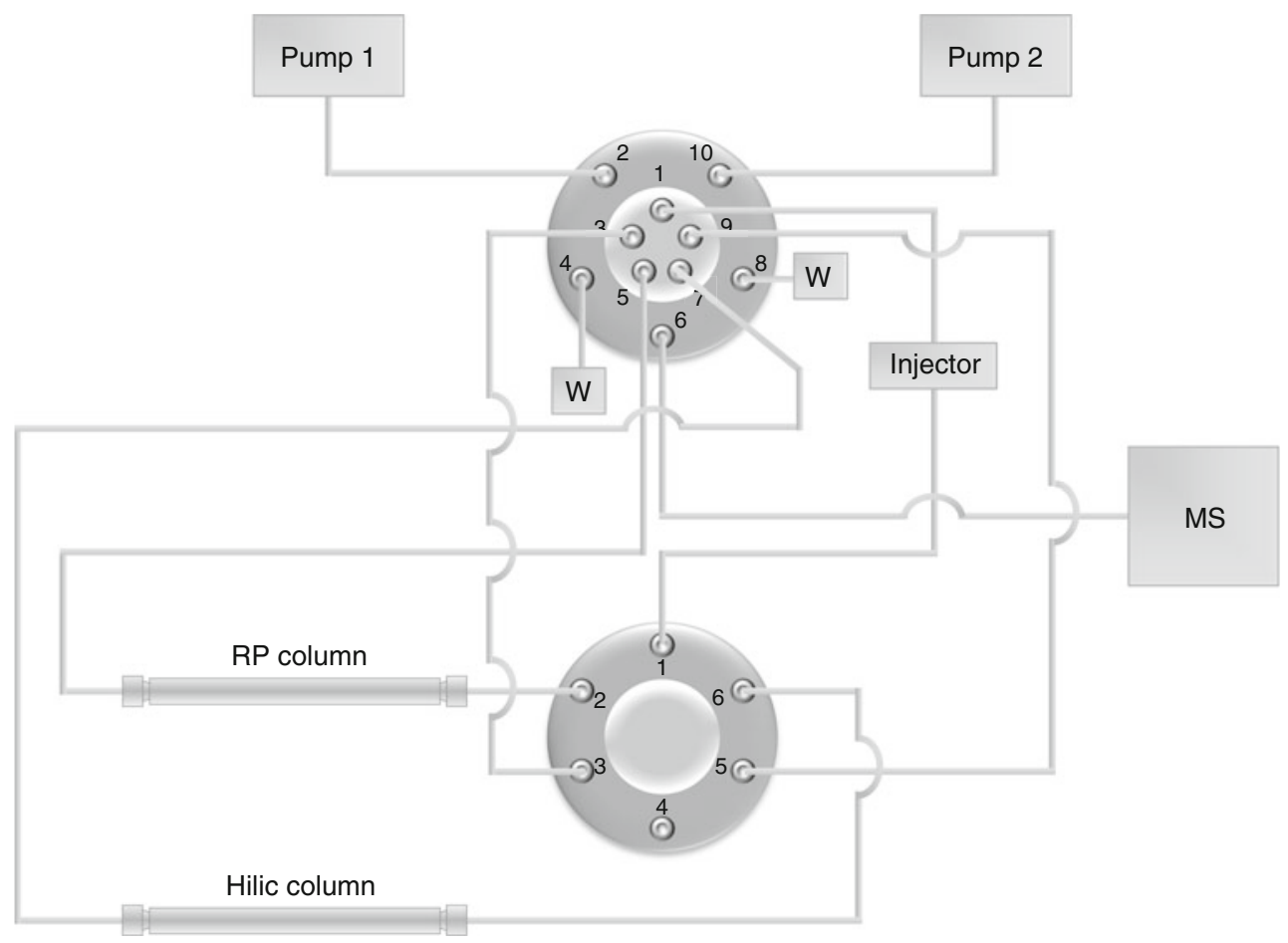

Fig. 1 HPLC setup featuring the two columns used in parallel. Pump 1 was linked to the RP column and pump 2 to the Hilic column. In RP mode, valves were in positions $1-2$ and $1-2$, and they were switched to positions $1-10$ and $1-6$ in Hilic mode 
sample on the RP (RP mode) and Hilic (Hilic mode) columns. Gradients applied on the two columns during both phases are described in Fig. 2. In RP mode, $\mathrm{ACN} / \mathrm{H}_{2} \mathrm{O}$ was set to $2 / 98 \mathrm{v} / \mathrm{v}$ for $0.1 \mathrm{~min}$ on the RP column and then brought to $90 / 10$ over $1.9 \mathrm{~min}$. These proportions were maintained for $2 \mathrm{~min}$, and the column was then flushed with $100 \%$ ACN. Meanwhile, the Hilic column was flushed with $\mathrm{ACN} / \mathrm{H}_{2} \mathrm{O} / \mathrm{FB}(50 / 48 / 2, \mathrm{v} / \mathrm{v} / \mathrm{v})$ for $1 \mathrm{~min}$, and then equilibrated to the initial conditions of the Hilic mode. In the Hilic mode, $\mathrm{ACN} / \mathrm{H}_{2} \mathrm{O} / \mathrm{FB}$ was set to $97 / 1 / 2$ for $0.5 \mathrm{~min}$ on the Hilic column and then brought to $60 / 38 / 2$ in $2.5 \mathrm{~min}$. These proportions were maintained for $1 \mathrm{~min}$, and then the column was flushed with 50/48/2. Meanwhile, the $\mathrm{RP}$ column was flushed with $100 \% \mathrm{ACN}$ for $1 \mathrm{~min}$ and then equilibrated to the RP mode initial conditions.

The Turbolon Spray interface was operated in the positive ionization mode. Nitrogen was used as the curtain and nebulizer gas, and the source parameters were set to a temperature of $650{ }^{\circ} \mathrm{C}$, a capillary voltage of $5,000 \mathrm{~V}$, an entrance potential of $10 \mathrm{~V}$, a collision cell exit potential of $10 \mathrm{~V}$, a curtain gas pressure of $20 \mathrm{psi}$, a nebulizer gas (GS1) pressure of $30 \mathrm{psi}$, and an auxiliary gas (GS2) pressure of 40 psi.

A data dependent acquisition (DDA) was defined to acquire the MS data as follows: selected reaction monitoring (SRM) transitions based on collision-induced dissociation (CID) occurring in the collision cell (quadrupole 2) were acquired. Then, 191 and 181 SRM transitions for the RP and the Hilic mode, respectively, were gathered from the method applied in our laboratory and from the iMethod ${ }^{\mathrm{TM}}$ Forensic LC/MS/MS Spectral Library (version 2.0) supplied by $A B$ Sciex. If the signal for one of the transitions reached a defined threshold $(5,000$ and 15,000 cps for the RP and the Hilic mode, respectively), an enhanced product ion (EPI) spectrum was acquired with collision energies of 40, 60 and $80 \mathrm{eV}$. To avoid missing co-eluted compounds, ions for which a mass spectrum was acquired were excluded $5 \mathrm{~s}$ after one occurrence.

\section{Detection and Identification}

A compound detection was accepted if a peak with a signalto-noise $(\mathrm{S} / \mathrm{N})$ ratio greater than three was observable in the corresponding SRM transition at the correct retention time. When an EPI spectrum was acquired for the detected compound, SmileMS (version 1.1; GeneBio, Geneva, Switzerland) was used to identify the compound present in the sample by comparing the acquired spectrum with a spectral database. The database used in this study was created by merging the iMethod ${ }^{\mathrm{TM}}$ Forensic LC/MS/MS Spectral Library (version 2.0) and spectra acquired on our instrument. The minimum match factor for identification was set to 50 .

Evaluation of the Method

The process efficiency was evaluated using the approach of Matuszewski et al. [27], with adaptations for the sample preparation. Three parameters were measured in triplicate for the 22 selected model compounds: the extraction recoveries were calculated by comparing the absolute analyte peak area of spiked DBS $(100 \mathrm{ng} / \mathrm{mL})$ to those obtained from the corresponding methanolic solution $(5 \mathrm{ng} / \mathrm{mL})$ introduced in wells containing blank DBS. Filter paper and the matrix effects were estimated by comparing the absolute analyte peak area of methanolic solutions introduced in wells containing blank filter paper disks and blank DBS, respectively, to those obtained from the corresponding methanolic solution. Finally, the overall analyte recoveries were measured by comparing the absolute analyte peak area of spiked DBS to those obtained from the corresponding methanolic solution.

The limits of detection (LOD) were evaluated by injecting extracts of spiked DBS with concentrations of 1 , $5,10,50$, and $100 \mathrm{ng} / \mathrm{mL}$ of each analyte. LOD was defined as the lowest concentration giving a peak, with a signal-to-noise ratio greater than 3 . The limits of identification (LOI) correspond to the lowest concentrations from which SmileMS was able to identify the compound.
Fig. 2 Gradients used on the two columns. Continuous and dashed lines represent the gradients applied on the RP and Hilic columns, respectively
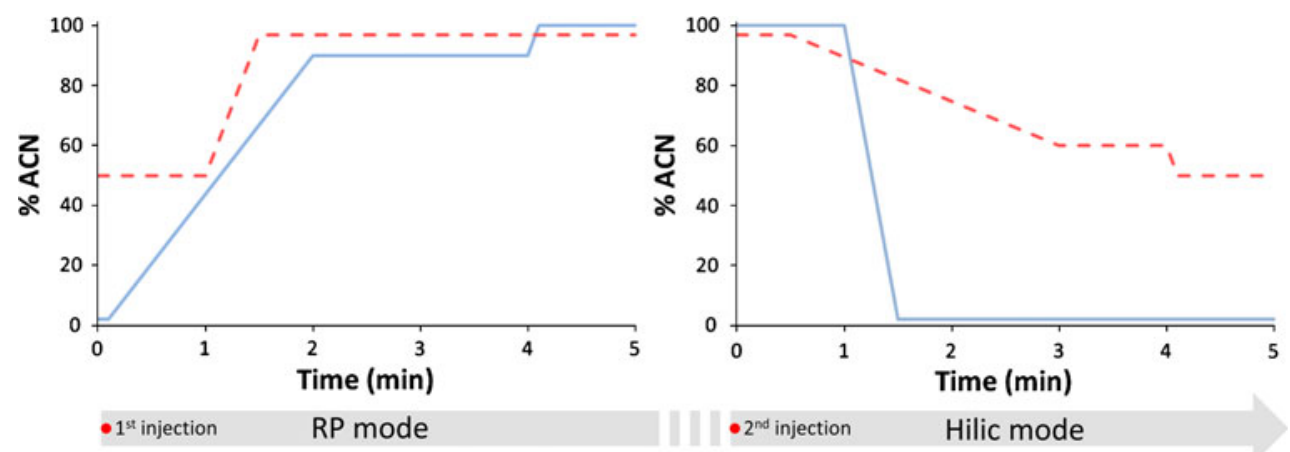
The described screening approach was then evaluated by quality control for benzodiazepines (Benzodiazepines plus 100 whole blood toxicology control) and pain management drugs (PM 100 (WB) whole blood toxicology control) from UTAK Laboratories (Valencia, USA), opioids substitution drugs (Medidrug ${ }^{\circledR}$ OSD 1/12-B VB) and drugs of abuse (Medidrug $^{\circledR}$ DOA-I VB low) from MEDICHEM Diagnostica (Steinenbronn, Germany), and 34 authentic postmortem samples received in our laboratories. The results obtained by LC-MS/MS were compared to those obtained during routine STA (i.e., GC-MS and LC-DAD) screening on whole blood $(1 \mathrm{~mL})$, as well as GC-MS(/MS) and LC-MS/MS quantitative analysis. Twenty different blank blood samples were also injected to evaluate the selectivity of the method, and carry-over was controlled by injecting blank $\mathrm{MeOH}$ after each sample injection.

\section{Results and Discussion}

\section{Sample Preparation}

In addition to its already well-known advantage of sample collection, DBS has also been described as a very promising technique to simplify sample preparation prior to analysis. Indeed, a simple soak of $5 \mu \mathrm{L}$ DBS in methanol proved to be sufficient to quantitatively and cleanly extract a class of drugs and quantify them within their therapeutic range [26]. This approach is all the more interesting for screening applications because methanol is a good solvent for a wide range of compounds, which makes this procedure theoretically compatible with many drug classes.

Extraction investigations showed that the automated procedure yielded extraction recoveries from 10 to $100 \%$, with a median extraction recovery of $40 \%$ (Table 1 ). This recovery was considered satisfactory regarding the fact that the extraction procedure was reduced to a minimum. No direct correlation between the extraction recovery and the hydrophilicity (expressed as $\log \mathrm{P}$ ) of the selected compounds could be observed, which indicated that this approach might be suitable for compounds with very different physico-chemical properties. For the compounds that were detectable in both chromatographic runs, the extraction recovery was noticed to be higher for the second injection. As already described [26], the extraction was not complete after $2 \mathrm{~min}$, suggesting that the extraction performance could be improved with an increased

Table 1 Matrix effects, extraction recoveries and overall process efficiencies for 22 model compounds, as estimated for the two chromatographic runs

\begin{tabular}{|c|c|c|c|c|c|c|}
\hline \multirow[t]{2}{*}{ Compound } & \multirow[t]{2}{*}{$\log P$} & \multicolumn{3}{|l|}{ Matrix effect } & \multirow{2}{*}{$\begin{array}{l}\text { Extraction recoveries } \\
\text { (RP/Hilic) }(\%)\end{array}$} & \multirow{2}{*}{$\begin{array}{l}\text { Overall process efficiency } \\
\text { (RP/Hilic) }(\%)\end{array}$} \\
\hline & & $\begin{array}{l}\text { Paper (RP/Hilic) } \\
(\%)\end{array}$ & $\begin{array}{l}\text { Blood (RP/Hilic) } \\
(\%)\end{array}$ & $\begin{array}{l}\text { Overall (RP/Hilic) } \\
(\%)\end{array}$ & & \\
\hline Cocaine & 3.08 & $114 / 106$ & $93 / 57$ & $107 / 60$ & $49 / 60$ & $52 / 36$ \\
\hline Cocaethylene & 2.79 & $110 / 98$ & $89 / 62$ & $98 / 61$ & $47 / 58$ & $46 / 35$ \\
\hline Benzoylecgonine & 2.26 & $88 / 89$ & $133 / 85$ & $118 / 75$ & $34 / 10$ & $40 / 8$ \\
\hline EME & 0.13 & $-/ 73$ & $-/ 21$ & $-/ 15$ & $-/ 77$ & $-/ 12$ \\
\hline Amphetamine & 1.81 & $-/ 98$ & $-/ 53$ & $-/ 52$ & $-/ 63$ & $-/ 33$ \\
\hline MDMA & 2.05 & $-/ 96$ & $-/ 30$ & $-/ 28$ & -174 & $-/ 21$ \\
\hline Methamphetamine & 2.20 & $-/ 10$ & $-/ 22$ & $-/ 22$ & $-/ 105$ & $-/ 23$ \\
\hline Morphine & 0.87 & $114 /-$ & $64 /-$ & $73 /-$ & $20 /-$ & $15 /-$ \\
\hline 6-MAM & 1.20 & $-/ 113$ & $-/ 103$ & $-/ 117$ & -138 & -145 \\
\hline Morphine-Glu & -1.56 & $-/ 70$ & $-/ 115$ & -180 & $-/ 17$ & $-/ 14$ \\
\hline Codeine & 1.20 & $-/ 125$ & $-/ 25$ & $-/ 31$ & -140 & $-/ 12$ \\
\hline Methadone & 3.93 & $107 / 99$ & $93 / 92$ & $100 / 91$ & $36 / 56$ & $35 / 51$ \\
\hline Diazepam & 2.80 & $110 /-$ & 83/- & $91 /-$ & $42 /-$ & $39 /-$ \\
\hline 7-Aminoclonazepam & 1.29 & $87 /-$ & $121 /-$ & $105 /-$ & $25 /-$ & $26 /-$ \\
\hline Midazolam & 3.80 & 99/- & $97 /-$ & $96 /-$ & $48 /-$ & $46 /-$ \\
\hline Hydroxymidazolam & 2.50 & 99/- & $106 /-$ & $105 /-$ & $40 /-$ & $42 /-$ \\
\hline Zolpidem & 4.41 & $94 /-$ & $95 /-$ & $89 /-$ & $35 /-$ & $31 /-$ \\
\hline Amitriptyline & 3.48 & $96 /-$ & $95 /-$ & $91 /-$ & $32 /-$ & $29 /-$ \\
\hline Citalopram & 4.71 & 93/- & 98/- & $91 /-$ & $42 /-$ & $39 /-$ \\
\hline Trimipramine & 3.94 & $96 / 101$ & $96 / 83$ & $93 / 84$ & $32 / 44$ & $30 / 37$ \\
\hline Clozapine & 3.76 & $103 /-$ & 88/- & $90 /-$ & $29 /-$ & $27 /-$ \\
\hline Haloperidol & 0.87 & $83 /-$ & $107 /-$ & $89 /-$ & $33 /-$ & $29 /-$ \\
\hline
\end{tabular}


extraction period. This was found to be unnecessary for this application.

Matrix effect was also evaluated, taking into account the effect of the filter paper and the blood (Table 1). No or minor effects were found when only clean filter paper was introduced into the methanolic solution of the selected model compounds, suggesting that no particular trapping effect or release of interfering compounds from the paper occurred during the extraction procedure. The presence of blank matrix revealed that interfering compounds extracted from blood impaired the detection of the compounds of interest. However, this effect was noticeable only when injecting on the Hilic column, which retained molecules with polar moieties. Consequently, a purification step as well as a more specific chromatographic gradient would have been necessary to eliminate phospholipids and other amphiphiles or polar interferences if a quantitative application was desired. For qualitative purposes, these matrix effects were found to be acceptable, as long as they did not dramatically impair the sensitivity.

The injection of blank methanol after the analysis of authentic samples showed no carry-over. This was also observable in $\mathrm{MeOH}$ injected after samples containing high concentrations of methadone $(710 \mathrm{ng} / \mathrm{mL})$, sertraline $(1,600 \mathrm{ng} / \mathrm{mL})$ and maprotiline $(6,900 \mathrm{ng} / \mathrm{mL})$, which presented no remaining peaks in the corresponding transitions. This proved that the rinsing of the syringe after the extraction step and the flushing of the columns after the chromatographic runs were satisfactory. Carry-over was low also because the extract was not pre-concentrated during the extraction procedure, which avoided injecting very high concentrations of drugs into the analytical system.

\section{Dual LC}

The major difficulty when developing a screening procedure is to cover the wide range of compounds that may be encountered in toxicological investigations as completely as possible. Due to the variety of drug structures and chemical properties, the emergence of a one-shot universal screening procedure is elusive. Column switching setups with orthogonal phases were proposed to expand the number of drugs amenable to separation, but these systems required long and complex chromatographic runs to enable satisfactory resolution.

The strategy that we have adopted in our laboratory was to reduce the analytical runs to increase throughput. We chose to set up two chromatographic columns with orthogonal phases in parallel (Fig. 1) to broaden the analytical capabilities of our system while keeping the chromatographic gradients very short and generic. Moreover, making two injections reduced the post-analysis time, as reconditioning of one column could be conducted while a separation was running on the other (Fig. 2). This approach is particularly interesting for screening both nonpolar compounds, such as benzodiazepines, and polar compounds, such as amphetamines, which do not respond well to RP conditions, or metabolites, such as glucuronides. This system was found to be stable, as standard deviations of 0.31 and $0.51 \%$ over 52 injections were measured on the RP and Hilic columns, respectively, for $\mathrm{CS}$ retention time.

Table 2 shows that most of the compounds present in different commercial controls covering frequently encountered drug families were detectable in the RP run under the described chromatographic conditions, provided the SRM transition was in the method. In this way, $67 \%$ of the compounds were visible in the first run. On the second run, only $28 \%$ of the compounds were detected. This discrepancy was due to the bad chromatographic behavior of the very nonpolar benzodiazepines on this phase. Nevertheless, amphetamines were detectable only on the Hilic column.

Therefore, the use of two columns with orthogonal phases allowed for detection of $80 \%$ of the drugs present in the controls for which a SRM transition was known. However, the method did not allow the detection of cannabinoids, mainly due to their very low concentrations in the controls and bad responses to the MS conditions.

\section{Detection and Identification}

The use of DBS is limited by the sensitivity of the instrument. The volume of the DBS sample is very low, and the analytes cannot be pre-concentrated during the sample preparation step described in this work. MS/MS is then mandatory to lower the detection limits, as it enables a drastic reduction in the noise. However, even if tandem mass spectrometry is a very selective technique, a single SRM transition is not sufficient to formally identify the detected compounds. This is particularly true for multianalyte procedures in complex matrices, for which crossdetections and interferences cannot be exhaustively investigated. Moreover, methods with several SRM transitions for each compound become more difficult to manage as the number of targets increases. To bypass this problem, a DDA program was implemented that allowed combining the very high sensitivity of SRM transitions with the good specificity of EPI spectra.

Table 3 indicates the LOD and LOI for the 22 model compounds. These two values may differ because LOD is based on the signal-to-noise ratio, whereas the LOI is based on the peak intensity. The LOI is then directly linked to the threshold set in the DDA program to acquire the EPI spectra, and may be lowered if required. In the conditions that were used for this work, LOD and LOI were found to be satisfactory in comparison with the therapeutic ranges found in the literature [28] or the positivity thresholds 
Table 2 Detection and identification of substances present in four commercial quality controls

\begin{tabular}{|c|c|c|c|}
\hline Compound & Concentrations (ng/mL) & Retention time (min, RP/Hilic) & Identification \\
\hline \multicolumn{4}{|c|}{ UTAK Benzodiazepines plus 100 whole blood toxicology control } \\
\hline Alprazolam & 100 & $2.60 /-$ & $\boldsymbol{V}$ \\
\hline Hydroxyalprazolam & 100 & $2.54 /-$ & $\times$ \\
\hline Bromazepam & 100 & - & $x$ \\
\hline Chlordiazepoxyde & 100 & $2.68 /-$ & $\times$ \\
\hline Clobazam & 100 & $2.74 /-$ & $\times$ \\
\hline Clonazepam & 100 & $2.64 /-$ & $\checkmark$ \\
\hline 7-Aminoclonazepam & 100 & $2.30 /-$ & $\checkmark$ \\
\hline Diazepam & 100 & $2.87 /-$ & $\checkmark$ \\
\hline Nordiazepam & 100 & $2.71 /-$ & $\times$ \\
\hline Estazolam & 100 & Transition missing & $\times$ \\
\hline Flunitrazepam & 100 & $2.71 /-$ & $\checkmark$ \\
\hline 7-Aminoflunitrazepam & 100 & $2.38 /-$ & $x$ \\
\hline Flurazepam & 100 & $2.88 /-$ & $\checkmark$ \\
\hline 2-Hydroxyethylflurazepam & 100 & Transition missing & $\times$ \\
\hline Lorazepam & 100 & $2.60 /-$ & $\times$ \\
\hline Lormetazepam & 100 & $2.74 /-$ & $\checkmark$ \\
\hline Midazolam & 100 & $2.81 /-$ & $\checkmark$ \\
\hline Hydroxymidazolam & 100 & $2.63 /-$ & $\checkmark$ \\
\hline Nitrazepam & 100 & $2.60 /-$ & $\times$ \\
\hline 7-Aminonitrazepam & 100 & $2.27 /-$ & $\times$ \\
\hline Oxazepam & 100 & $2.57 /-$ & $\checkmark$ \\
\hline Prazepam & 100 & $3.11 /-$ & $\checkmark$ \\
\hline Temazepam & 100 & $2.69 /-$ & $\checkmark$ \\
\hline Triazolam & 100 & $2.62 /-$ & $\times$ \\
\hline Hydroxytriazolam & 100 & Transition missing & \\
\hline Zolpidem & 100 & $2.65 /-$ & $\checkmark$ \\
\hline \multicolumn{4}{|l|}{ Medidrug ${ }^{\circledR}$ DOA-I VB low } \\
\hline Amphetamine & 11 & $-/ 3.41$ & $\checkmark$ \\
\hline MDA & 15 & $-/ 2.85$ & $\times$ \\
\hline MDE & 16 & $-/ 2.80$ & $\checkmark$ \\
\hline MDMA & 16 & $-/ 2.89$ & $\boldsymbol{V}$ \\
\hline Methamphetamine & 16 & $-/ 2.89$ & $\times$ \\
\hline MBDB & 21 & Transition missing & \\
\hline Cocaine & 10 & $2.68 / 2.93$ & $\checkmark$ \\
\hline Benzoylecgonine & 16 & $2.10 /-$ & $\checkmark$ \\
\hline Egonine methyl ester & 16 & -13.26 & $\checkmark$ \\
\hline Morphine & 11 & - & $\times$ \\
\hline Codeine & 22 & - & $\times$ \\
\hline Dihydrocodeine & 21 & - & $\times$ \\
\hline $\mathrm{THC}$ & 1 & - & $\times$ \\
\hline 11-OH-THC & 2 & - & $\times$ \\
\hline THC-COOH & 10 & - & $\times$ \\
\hline Cocaethylene & 15 & $2.82 / 2.90$ & $\checkmark$ \\
\hline \multicolumn{4}{|l|}{ Medidrug $^{\circledR}$ OSD 1/12-B VB } \\
\hline Buprenorphine & 5 & - & $\times$ \\
\hline Norbuprenorphine & 8 & - & $\times$ \\
\hline Methadone & 100 & $3.20 / 2.72$ & $\checkmark$ \\
\hline EDDP & 30 & $3.13 / 2.75$ & $\checkmark$ \\
\hline
\end{tabular}


Table 2 continued

\begin{tabular}{|c|c|c|c|}
\hline Compound & Concentrations (ng/mL) & Retention time (min, RP/Hilic) & Identification \\
\hline \multicolumn{4}{|c|}{ UTAK PM 100 (WB) whole blood toxicology control } \\
\hline Buprenorphine & 10 & - & $\times$ \\
\hline Norbuprenorphine & 10 & - & $\times$ \\
\hline Codeine & 100 & $2.10 /-$ & $\checkmark$ \\
\hline Fentanyl & 10 & $2.88 / 2.76$ & $\checkmark$ \\
\hline Norfentanyl & 10 & Transition missing & \\
\hline Hydrocodone & 100 & $2.31 /-$ & $\checkmark$ \\
\hline Hydromorphone & 100 & $1.96 /-$ & $\checkmark$ \\
\hline Meperidine & 100 & $3.20 / 2.73$ & $\checkmark$ \\
\hline Methadone & 100 & $3.14 / 2.76$ & $\checkmark$ \\
\hline EDDP & 100 & $3.14 / 2.76$ & $\checkmark$ \\
\hline Morphine & 100 & $1.95 /-$ & $x$ \\
\hline Oxycodone & 100 & $2.46 /-$ & $\checkmark$ \\
\hline Oxymorphone & 100 & - & $x$ \\
\hline Tapentadol & 100 & Transition missing & \\
\hline Tramadol & 100 & $2.53 / 2.82$ & $\checkmark$ \\
\hline
\end{tabular}

Table 3 Limits of detection (LOD) and identification (LOI) estimated for 22 model compounds

\begin{tabular}{|c|c|c|c|}
\hline Compound & $\begin{array}{l}\text { LOD } \\
(\mathrm{ng} / \mathrm{mL})\end{array}$ & $\begin{array}{l}\text { LOI } \\
(\mathrm{ng} / \mathrm{mL})\end{array}$ & $\begin{array}{l}\text { Therapeutic } \\
\text { range-legal } \\
\text { threshold } \\
(\mathrm{ng} / \mathrm{mL})\end{array}$ \\
\hline Cocaine & $<1$ & 1 & 15 \\
\hline Cocaethylene & $<1$ & 1 & - \\
\hline Benzoylecgonine & 1 & 5 & - \\
\hline EME & 10 & 10 & - \\
\hline Amphetamine & 1 & 10 & 15 \\
\hline MDMA & 1 & 10 & 15 \\
\hline Methamphetamine & 10 & 50 & 15 \\
\hline Morphine & 100 & $>100$ & 15 (free form) \\
\hline 6-MAM & 5 & 50 & - \\
\hline Morphine-Glu & 10 & 50 & - \\
\hline Codeine & 50 & 100 & $30-250$ \\
\hline Methadone & $<1$ & 1 & $100-500$ \\
\hline Diazepam & 50 & 50 & $200-2,000$ \\
\hline 7-Aminoclonazepam & 100 & 100 & - \\
\hline Midazolam & $<1$ & 5 & $40-100$ \\
\hline Hydroxymidazolam & 5 & 5 & - \\
\hline Zolpidem & $<1$ & 1 & $80-150$ \\
\hline Amitriptyline & $<1$ & 5 & $50-300$ \\
\hline Citalopram & $<1$ & 5 & $10-200$ \\
\hline Trimipramine & $<1$ & 1 & $10-250$ \\
\hline Clozapine & $<1$ & 5 & $300-600$ \\
\hline Haloperidol & $<1$ & 1 & $5-20$ \\
\hline
\end{tabular}

defined by the Swiss authorities [29], except for morphine. This compound was difficult to detect due to its incomplete extraction, the presence of matrix effect in the Hilic mode and its poor chromatographic performance in the RP phase.

This approach is limited by the number of available SRM transitions but enables the detection of most compounds of toxicological interest. Another limitation is that the cycle time of DDA is relatively long (1.3702 and $1.3101 \mathrm{~s}$ for the RP and Hilic runs, respectively) in comparison with the peak widths ( 3 to $20 \mathrm{~s}$, depending on the compound). This means that the system is not able to acquire a mass spectrum if the peak is not at least three times as large as the cycle time, which corresponds to two acquisition points, and cannot acquire a mass spectrum for coeluted compounds if the peak widths are not sufficient. This is the reason why not all the benzodiazepines in Table 2 could be identified by Smile MS even when a SRM signal was perfectly defined. However, the concomitant presence of 25 benzodiazepines in one real case sample is not likely to happen. More generally, the decision to exclude identified compounds in the DDA program can be made by a toxicologist to focus on compounds for which no EPI spectrum can be acquired. This strategy is all the more reasonable because the entire screening procedure lasts only $12 \mathrm{~min}$.

\section{Application to Authentic Samples}

To evaluate the method, 20 different blank blood samples and 33 authentic post-mortem cases were analyzed using 


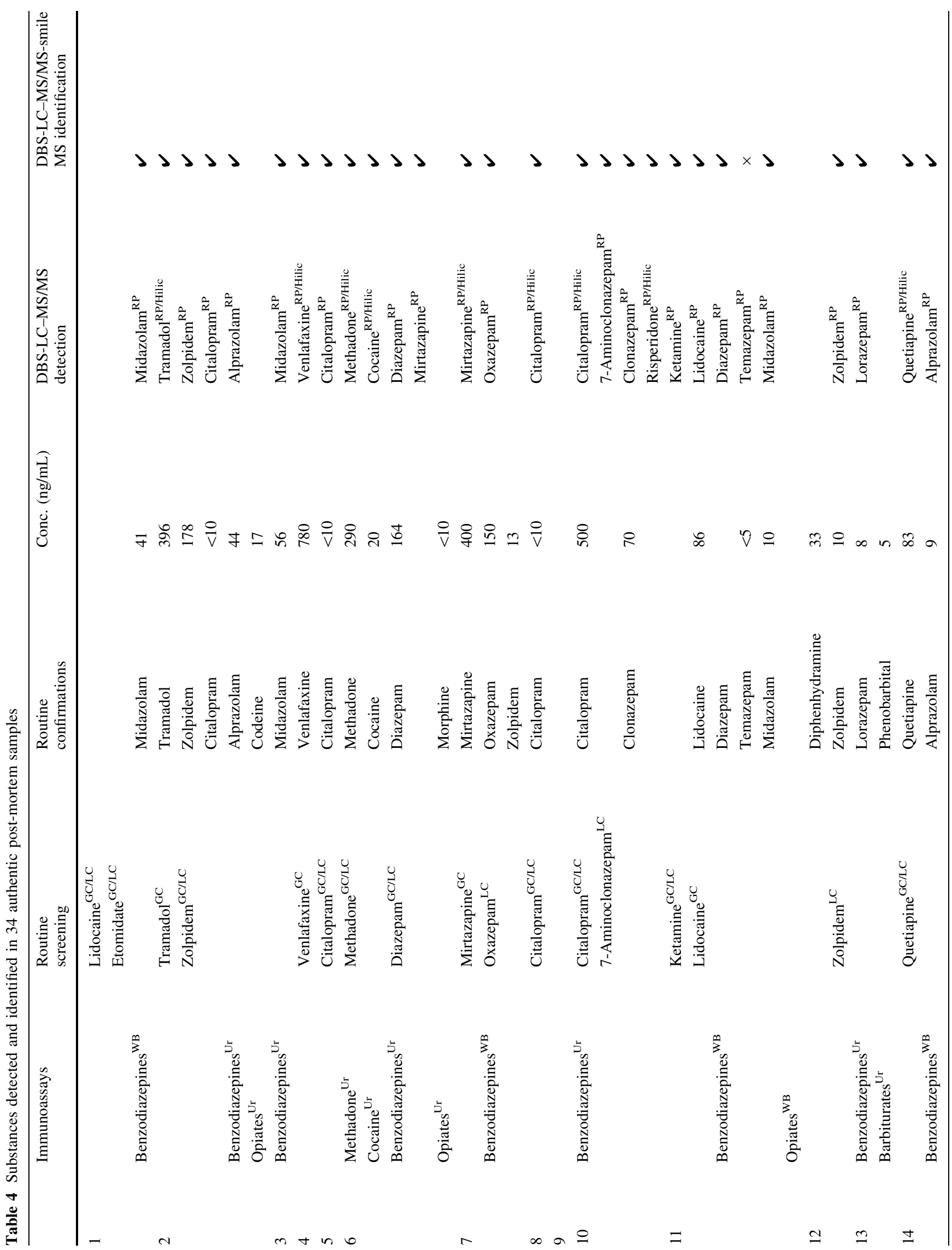




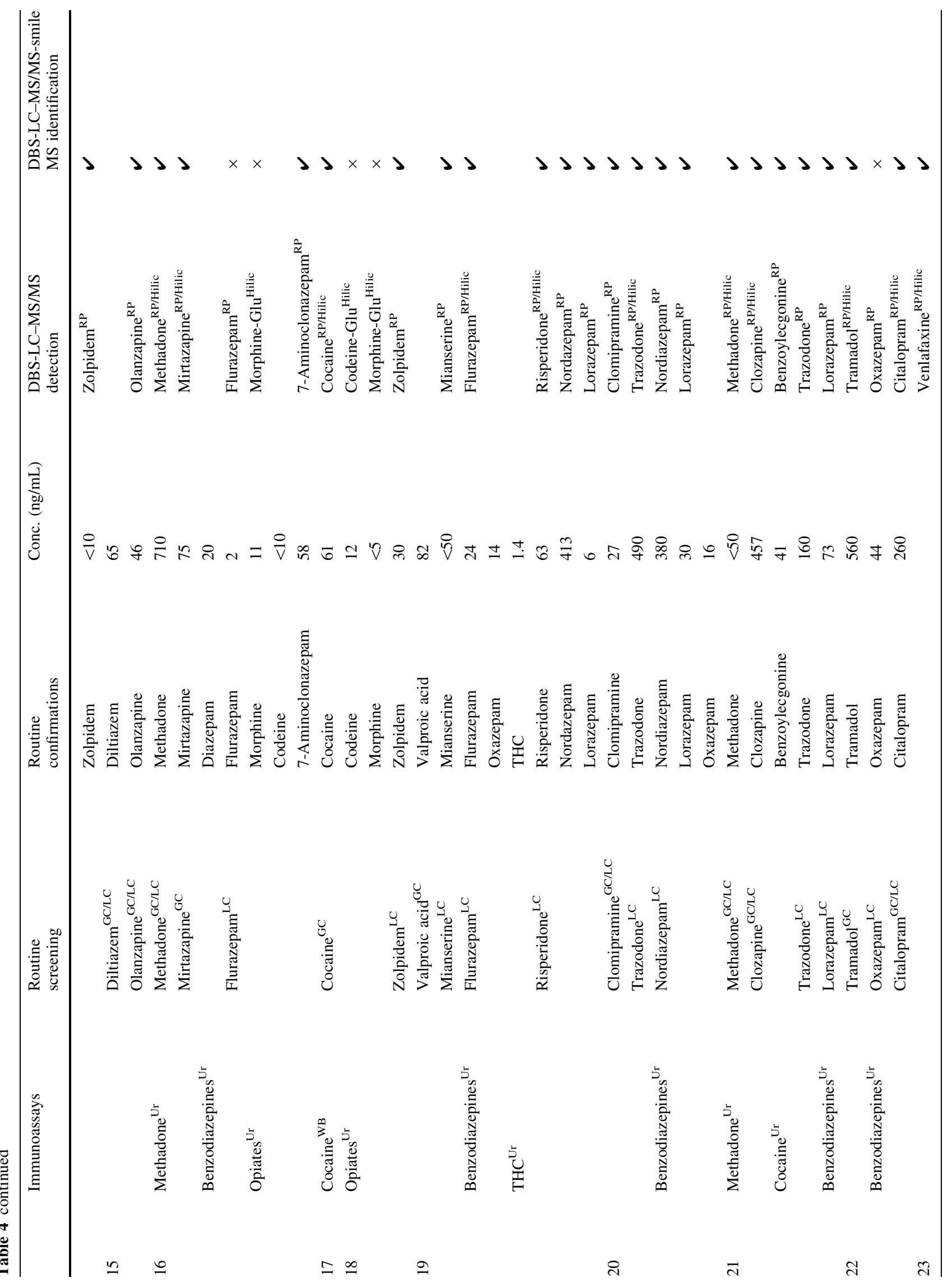




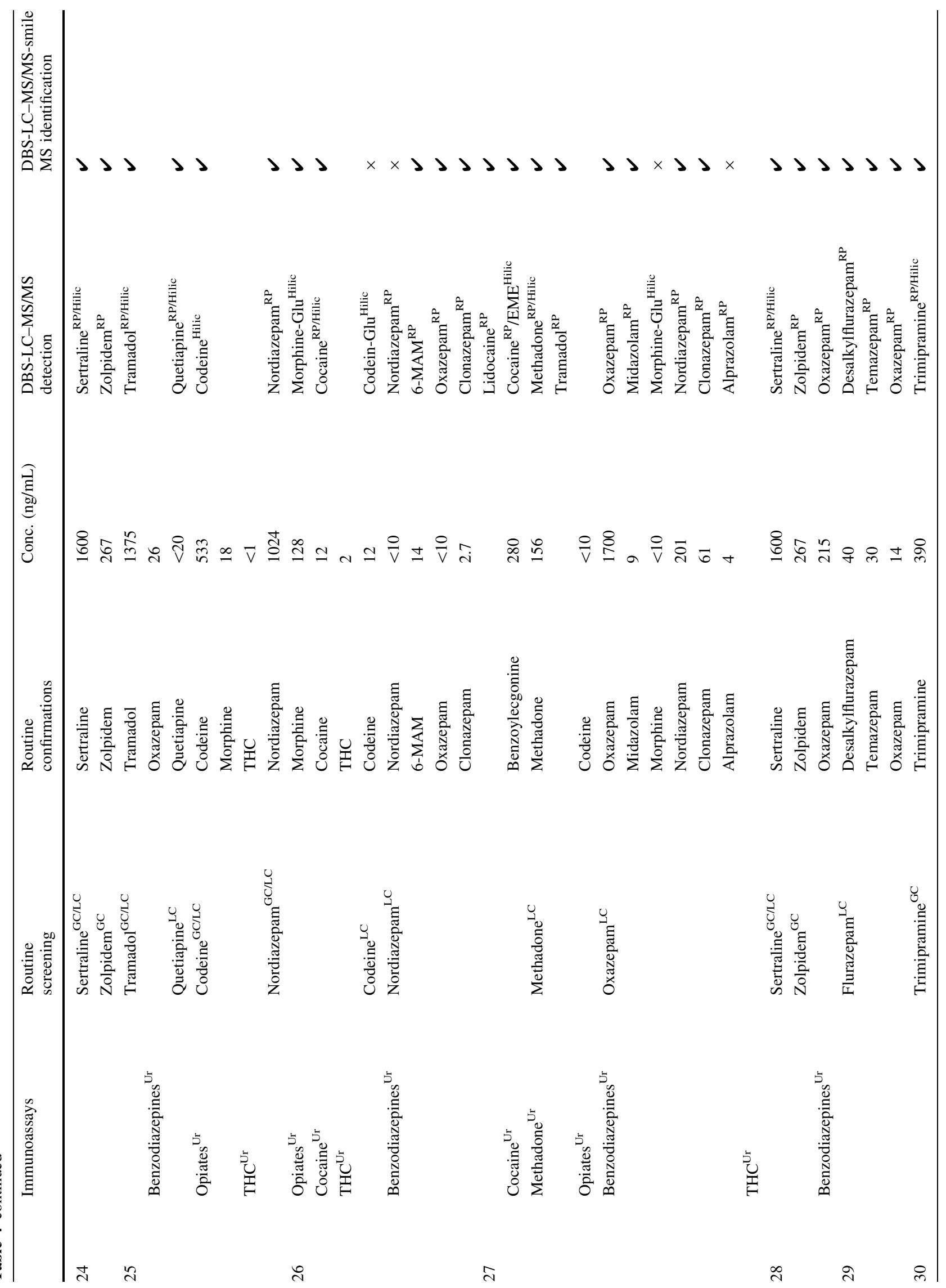




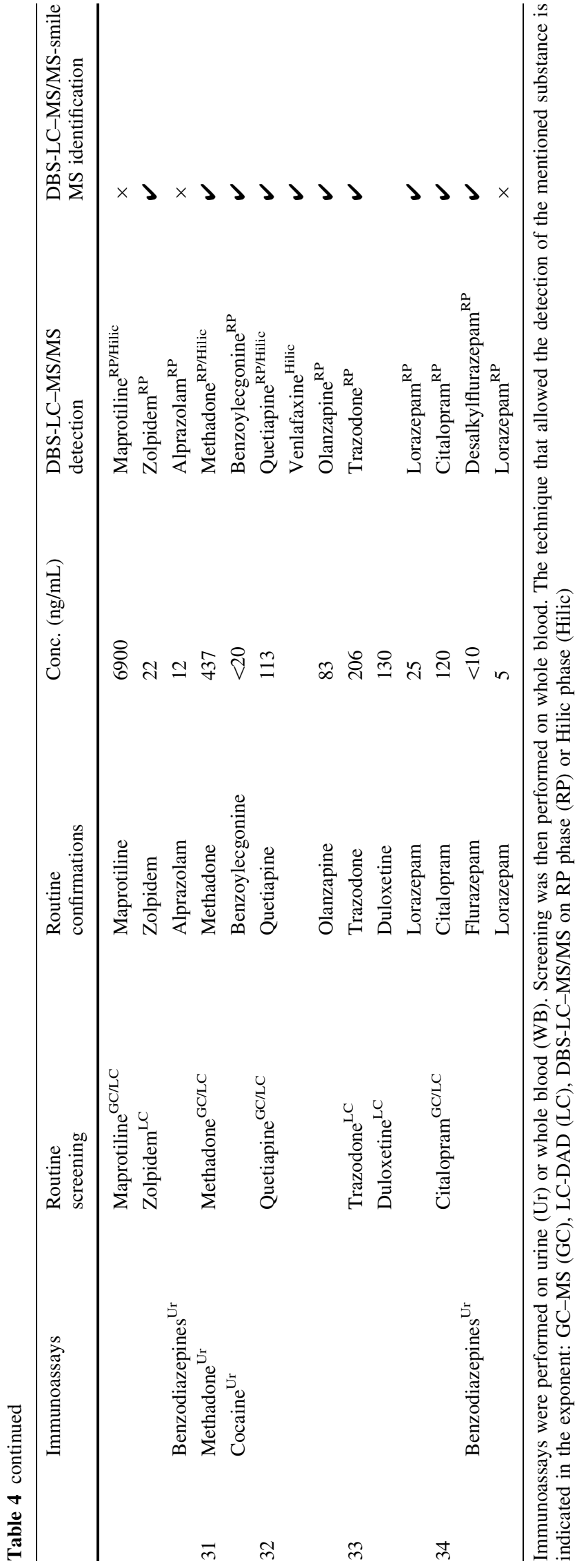

the method described in this work. No false positive match was observed in the 20 blank samples, indicating good selectivity of the screening method. The results from screening authentic post-mortem samples are presented in Table 4. To simplify the results table, the detection of metabolites is mentioned only if the parent drug was not detected. Moreover, substances of lower toxicological interest, such as caffeine or nicotine, are not mentioned.

This study showed that the DBS-LC-MS/MS target screening approach provided very satisfactory results compared to routine screening procedures, even if the sample volume was reduced from 1,000 to $5 \mu \mathrm{L}$. Specifically, routine screening enabled the detection of $55 \%$ of the drugs that were finally quantified, whereas the presented method allowed identification of $77 \%$ of them. This number was elevated to $89 \%$, if we also considered the SRM detections that did not trigger the EPI acquisition. Some compounds could not be detected due to missing SRM transitions (diltiazem and duloxetine), which illustrated the limits of the target screening approach. Some compounds were also not compatible with the analytical approach presented in this work (valproic acid and phenobarbital). Morphine and codeine were frequently missed, and cannabinoids could not be detected in any sample, as also observed for routine methods.

\section{Conclusion}

A rapid target screening procedure for forensic and clinical applications was proposed in this report. The use of DBS sampling allowed for the development of a very fast and simple sample preparation method that maintained good qualitative analysis performance. This step was easily automated without a dedicated instrument, which makes this approach easily implementable in any toxicological laboratory. The chromatographic setup presented in this article enabled increased separation capability while maintaining very short gradients and good chromatographic stability. Finally, very good detection and identification were observed due to the combination of the sensitivity of the SRM transitions and the very high specificity of the EPI spectra. This procedure proved to be a very powerful tool for forensic and clinical STA.

This method did show some limitations, particularly for morphine and cannabinoids, which were difficult to detect. Moreover, some compounds were not detected, as their SRM transitions were not known. This latter aspect was inherent to the target screening approach. Finally, the coupling of the sample preparation and dual LC separation methods to a more general detection and identification procedure, including high resolution MS, is perfectly feasible. 


\section{References}

1. Systematic toxicological analysis: laboratory guidelines (2012) The International Association of Forensic Toxicologists. http://www.tiaft.org/node/82. Accessed 24 Oct 2012

2. Vindenes V, Lund HME, Andresen W, Gjerde H, Ikdahl SE, Christophersen AS, Øiestad EL (2012) Detection of drugs of abuse in simultaneously collected oral fluid, urine and blood from Norwegian drug drivers. Forensic Sci Int 219:165-171

3. Viette V, Fathi M, Rudaz S, Hochstrasser D, Veuthey J-L (2011) Current role of liquid chromatography coupled to mass spectrometry in clinical toxicology screening methods. Clin Chem Lab Med 49:1091-1103

4. Parker SP, Cubitt WD (1999) The use of the dried blood spot sample in epidemiological studies. J Clin Pathol 52:633-639

5. Cartiser N, Bévalot F, Le Meur C, Gaillard Y, Malicier D, Hubert N, Guitton J (2011) Gas chromatography-tandem mass spectrometry assay for the quantification of four benzodiazepines and citalopram in eleven postmortem rabbit fluids and tissues, with application to animal and human samples. J Chromatogr B Analyt Technol Biomed Life Sci 879:2909-2918

6. Déglon J, Thomas A, Mangin P, Staub C (2012) Direct analysis of dried blood spots coupled with mass spectrometry: concepts and biomedical applications. Anal Bioanal Chem 402:2485-2498

7. Stove CP, Ingels A-SME, De Kesel PMM, Lambert WE (2012) Dried blood spots in toxicology: from the cradle to the grave? Crit Rev Toxicol 42:230-243

8. Thomas A, Geyer H, Schänzer W, Crone C, Kellmann M, Moehring T, Thevis M (2012) Sensitive determination of prohibited drugs in dried blood spots (DBS) for doping controls by means of a benchtop quadrupole/Orbitrap mass spectrometer. Anal Bioanal Chem 403:1279-1289

9. Versace F, Sporkert F, Mangin P, Staub C (2012) Rapid sample pre-treatment prior to GC-MS and GC-MS/MS urinary toxicological screening. Talanta 101:299-306

10. Peters FT (2011) Recent advances of liquid chromatography(tandem) mass spectrometry in clinical and forensic toxicology. Clin Biochem 44:54-65

11. Jandera P, Cesla P, Hájek T, Vohralík G, Vynuchalová K, Fischer J (2008) Optimization of separation in two-dimensional highperformance liquid chromatography by adjusting phase system selectivity and using programmed elution techniques. J Chromatogr A 1189:207-220

12. Thomas A, Déglon J, Steimer T, Mangin P, Daali Y, Staub C (2010) On-line desorption of dried blood spots coupled to hydrophilic interaction/reversed-phase LC/MS/MS system for the simultaneous analysis of drugs and their polar metabolites. J Sep Sci 33:873-879

13. Shushan BI, Marquet P, Dupuy JL, Anacleto J, Lachâtre G, Duchoslav E, Chan M (1999) Development of a toxicant screening procedure using liquid chromatography-electrospraymass-spectrometry (LC-ESMS). Ther Drug Monit 21:467

14. Marquet P, Venisse N, Lacassie E, Lachâtre G (2000) In-source CID mass spectral libraries for the "general unknown" screening of drugs and toxicants. Analusis 28:925-934

15. Marquet P (2002) Is LC-MS suitable for a comprehensive screening of drugs and poisons in clinical toxicology? Ther Drug Monit 24:125

16. Sturm S, Hammann F, Drewe J, Maurer HH, Scholer A (2010) An automated screening method for drugs and toxic compounds in human serum and urine using liquid chromatography-tandem mass spectrometry. J Chromatogr B 878:2726-2732

17. Sauvage F-L, Picard N, Saint-Marcoux F, Gaulier J-M, Lachâtre G, Marquet P (2009) General unknown screening procedure for the characterization of human drug metabolites in forensic toxicology: applications and constraints. J Sep Sci 32:3074-3083

18. Liu H-C, Liu RH, Lin D-L, Ho H-O (2010) Rapid screening and confirmation of drugs and toxic compounds in biological specimens using liquid chromatography/ion trap tandem mass spectrometry and automated library search. Rapid Commun Mass Spectrom 24:75-84

19. Mueller C, Weinmann W, Dresen S (2005) Development of a multi-target screening analysis for 301 drugs using a QTrap liquid chromatography/tandem mass spectrometry system and automated library searching. Rapid Commun Mass Spectrom 19: $1332-1338$

20. Viette V, Guillarme D, Mylonas R, Mauron Y, Fathi M, Rudaz S, Hochstrasser D, Veuthey J-L (2011) A multi-target screening analysis in human plasma using fast liquid chromatographyhybrid tandem mass spectrometry (Part I). Clin Biochem 44: $32-44$

21. Viette V, Guillarme D, Mylonas R, Mauron Y, Fathi M, Rudaz S, Hochstrasser D, Veuthey J-L (2011) A multi-target screening analysis in human plasma using fast liquid chromatographyhybrid tandem mass spectrometry (Part II). Clin Biochem 44: $45-53$

22. Thomas A, Hopfgartner G, Giroud C, Staub C (2009) Quantitative and qualitative profiling of endocannabinoids in human plasma using a triple quadrupole linear ion trap mass spectrometer with liquid chromatography. Rapid Commun Mass Spectrom 23:629-638

23. Mueller DM, Rentsch KM (2012) Online extraction toxicological MS(n) screening system for serum and heparinized plasma and comparison of screening results between plasma and urine in the context of clinical data. J Chromatogr B Analyt Technol Biomed Life Sci 883-884:189-197

24. McCauley-Myers DL, Eichhold TH, Bailey RE (2000) Rapid bioanalytical determination of dextromethorphan in canine plasma by dilute-and-shoot preparation combined with one minute per sample LC-MS/MS analysis to optimize formulations for drug delivery. J Pharmaceut Biomed 23:825-835

25. Li F, Ploch S (2012) Will "green" aspects of dried blood spot sampling accelerate its implementation and acceptance in the pharmaceutical industry? Bioanalysis 4:1259-1261

26. Déglon J, Versace F, Lauer E, Widmer C, Mangin P, Thomas A, Staub C (2012) Rapid LC-MS/MS quantification of the major benzodiazepines and their metabolites on dried blood spots using a simple and cost-effective sample pretreatment. Bioanalysis 4:1337-1350

27. Matuszewski BK, Constanzer ML, Chavez-Eng CM (2003) Strategies for the assessment of matrix effect in quantitative bioanalytical methods based on HPLC-MS/MS. Anal Chem 75:3019-3030

28. Iwersen-Bergmann S, Andresen H, Schmoldt A (2012) Therapeutic and toxic blood concentrations of nearly 1,000 drugs and other xenobiotics. Crit Care 16:R136

29. Ordonnance de l'OFROU concernant l'ordonnace sur le contrôle de la circulation routière, art. 34 (2012) The Federal Authorities of the Swiss Confederation. http://www.admin.ch/ch/f/rs/741_ 013_1/a34.html. Accessed 24 Oct 2012 